\title{
A Novel Wide-Band Noise-Parameter Measurement Method and Its Cryogenic Application
}

\author{
Robert Hu and Sander Weinreb, Life Fellow, IEEE
}

\begin{abstract}
The concept of using a long mismatched transmission line to measure noise parameters has been known for some time. However, it has been limited to narrow-bandwidth applications, and a wide-band extension has never been reported. In order to measure the cryogenic noise parameters of a wide-band low-noise amplifier (LNA), a wide-band frequency-variation method is proposed. In this method, the four noise parameters at each frequency are derived numerically from a set of matched and mismatched noise temperatures measured within a surrounding frequency-sampling window. By scanning this frequency-sampling window, noise parameters over a wide frequency range can be obtained. Since this approach can be easily incorporated into existing noise measurement systems, a tuner is not required, and the technique can be applied to a cryogenic amplifier. This paper details the theory, implementation, and verification of this new method. The measured noise parameters of a cryogenic wide-band LNA are presented.
\end{abstract}

Index Terms-Frequency-variation method, noise-parameter measurement.

\section{INTRODUCTION}

$\mathbf{T}$ HE cryogenic low-noise amplifier (LNA) is a critical component in sensitive receivers used in communications and radio astronomy. A particular case of interest is for wide-band LNAs following superconducting millimeter mixers without using a bandwidth-limiting isolator [1]-[3]. The mixer's output impedance is dependent upon local-oscillator (LO) frequency and power, and the cryogenic noise parameters of the following LNA must be known to optimize the receiver noise. In this paper, a novel wide-band frequency-variation method is proposed to facilitate this kind of measurement. It is also found that this new method works equally well at room temperature; therefore, it can be used as a substitute for the more expensive and complicated tuner method [4].

In Section II, prior to the formal introduction of the numerical wide-band frequency-variation method, the least squares fit and analytical narrow-band frequency-variation method are first discussed. As is commonly used in tuner-based noise-parameter measurement systems, the method of least squares fit provides the necessary mathematical foundation. By combining this numerical approach with the narrow-band frequency-variation concept, a wide-band frequency-variation method is proposed.

In Section III, a simulation is carried out to verify the newly proposed method. The simulated results quantify the tradeoff

Manuscript received November 21, 2003; revised January 18, 2004.

R. Hu is with Academia Sinica, Taipei 106, Taiwan, R.O.C.

$\mathrm{S}$. Weinreb is with the Department of Electrical Engineering, California Institute of Technology, Pasadena, CA 91125 USA.

Digital Object Identifier 10.1109/TMTT.2004.827029 between unwanted ripples and frequency smearing of the noise parameters, as determined by the width and weighting of a frequency window. Following an error analysis of the generator impedance, a reactive circuit realizing the desired impedance over a wide frequency range is described.

Section IV presents experimental verification of the wide-band frequency-variation method. To demonstrate the accuracy of this method, the noise parameters of a reciprocal passive circuit are measured and compared with the values determined by $S$-parameter measurements. As a further demonstration, the noise parameters of a room-temperature LNA measured with both the wide-band frequency-variation method and the conventional tuner method are compared.

The application of the wide-band frequency-variation method in cryogenic noise-parameter measurement is illustrated in Section V. It shows how this method can be incorporated into the cryogenic noise measurement setups, which use the availability of cryogenic terminations to increase the measurement accuracy of sensitive LNAs. Finally, the measured noise parameters of a cryogenic wide-band LNA are presented.

\section{TheORY OF Wide-BAND FREQUENCY-VARIaTION METHOD}

\section{A. Least Squares Fit for Noise-Parameter Calculation}

In the conventional tuner method, the least-squares fit can be used to derive the noise parameters from a set of generator reflection coefficients and their corresponding noise temperatures. The key point is the linearization of the noise temperature expression [5]. First, the noise temperature is expressed as a function of four noise parameters [6]

$$
T_{n}=T_{\min }+4 T_{0} N \frac{\left|\Gamma_{g}-\Gamma_{\mathrm{opt}}\right|^{2}}{\left(1-\left|\Gamma_{g}\right|^{2}\right)\left(1-\left|\Gamma_{\mathrm{opt}}\right|^{2}\right)}
$$

where $\Gamma_{g}\left(=\gamma_{g} \exp \left(j \theta_{g}\right)\right)$ is the generator reflection coefficient and $T_{0}$ is $290 \mathrm{~K}$. The four noise parameters $T_{\min }, N$ and complex $\Gamma_{\text {opt }}\left(=\gamma_{\text {opt }} \exp \left(j \theta_{\text {opt }}\right)\right)$ are the minimum noise temperature, noise ratio, and optimum generator reflection coefficient, respectively. Hence, not just $T_{\min }$, but $N$ too, will be invariant under a reciprocal lossless transformation like the case of a lossless input transmission line or inductor. The relation between the unitless $N$ and the more commonly used noise resistance $R_{n}$ is $N=R_{n} \cdot \operatorname{Re}\left[1 / Z_{\text {opt }}\right]$ with $Z_{\text {opt }}$ being the optimum generator impedance.

The above $T_{n}$ expression can now be transformed into a linear function of another four variables $a, b, c, d$ as follows:

$$
T_{n}=a+b \frac{1}{1-\gamma_{g}^{2}}+c \frac{\gamma_{g} \cos \left(\theta_{g}\right)}{1-\gamma_{g}^{2}}+d \frac{\gamma_{g} \sin \left(\theta_{g}\right)}{1-\gamma_{g}^{2}}
$$


with

$$
\begin{aligned}
& a=-4 T_{0} N \frac{1}{1-\gamma_{\mathrm{opt}}^{2}}+T_{\mathrm{min}} \\
& b=4 T_{0} N \frac{1+\gamma_{\mathrm{opt}}^{2}}{1-\gamma_{\mathrm{opt}}^{2}} \\
& c=-8 T_{0} N \frac{\gamma_{\mathrm{opt}} \cos \left(\theta_{\mathrm{opt}}\right)}{1-\gamma_{\mathrm{opt}}^{2}} \\
& d=-8 T_{0} N \frac{\gamma_{\mathrm{opt}} \sin \left(\theta_{\mathrm{opt}}\right)}{1-\gamma_{\mathrm{opt}}^{2}} .
\end{aligned}
$$

Or, inversely, by setting $\Delta=\sqrt{b^{2}-c^{2}-d^{2}}$, we obtain

$$
\begin{aligned}
T_{\text {min }} & =a+\frac{b+\Delta}{2} \\
N & =\frac{\Delta}{4 T_{0}} \\
\gamma_{\mathrm{opt}} & =\sqrt{\frac{b-\Delta}{b+\Delta}} \\
\theta_{\mathrm{opt}} & =\tan ^{-1}\left(\frac{d}{c}\right) .
\end{aligned}
$$

At each frequency, the generator reflection coefficients $\Gamma_{i}\left(=\gamma_{i} \exp \left(j \theta_{i}\right)\right)$ and the corresponding noise temperatures $T_{i}$ with $i=1 \sim m$ can be arranged as

$$
\left[\begin{array}{c}
T_{1} \\
T_{2} \\
\vdots \\
T_{m}
\end{array}\right]=\left[\begin{array}{cccc}
1 & \frac{1}{1-\gamma_{1}^{2}} & \frac{\gamma_{1} \cos \left(\theta_{1}\right)}{1-\gamma_{1}^{2}} & \frac{\gamma_{1} \sin \left(\theta_{1}\right)}{1-\gamma_{1}^{2}} \\
1 & \frac{1}{1-\gamma_{2}^{2}} & \frac{\gamma_{2} \cos \left(\theta_{2}\right)}{1-\gamma_{2}^{2}} & \frac{\gamma_{2} \sin \left(\theta_{2}\right)}{1-\gamma_{2}^{2}} \\
\vdots & \vdots & \vdots & \vdots \\
1 & \frac{1}{1-\gamma_{m}^{2}} & \frac{\gamma_{m} \cos \left(\theta_{m}\right)}{1-\gamma_{m}^{2}} & \frac{\gamma_{m} \sin \left(\theta_{m}\right)}{1-\gamma_{m}^{2}}
\end{array}\right]\left[\begin{array}{l}
a \\
b \\
c \\
d
\end{array}\right]
$$

or

$$
[T]=[X][A]
$$

By applying the least squares fit, we obtain

$$
[A]=\left([X]^{t}[X]\right)^{-1}[X]^{t}[T] \text {. }
$$

To have a convergent solution, certain $\Gamma_{g}$ loci should be avoided [7]. One is a circle on the Smith chart where all the $\gamma_{g}$ will be the same; therefore, the second column of $[X]$ is a multiple of its first column. The other is a straight line on the Smith chart where the constant $\theta_{g}$ will force the third and fourth columns of $[X]$ to differ only by a constant factor of $\cos \left(\theta_{g}\right) / \sin \left(\theta_{g}\right)$.

\section{B. Analytical Narrow-Band Frequency-Variation Method}

The idea of a narrow-band frequency-variation method is similar to that of a standing wave. By inserting a cable between the mismatched noise source and LNA, the periodic $\Gamma_{g}$ results in a noise-temperature curve like a standing wave on the frequency axis. Assumed to be frequency independent, the four noise parameters can then be derived from the matched and mismatched noise temperatures [8], [9]. Intuitively, for a periodic $\Gamma_{g}$, the corresponding mismatched noise temperature curve can be characterized by three variables, i.e., magnitude, phase, and average value. These three values, together with the matched noise temperature, constitute the four constraints necessary for solving the four unknowns (Fig. 1).
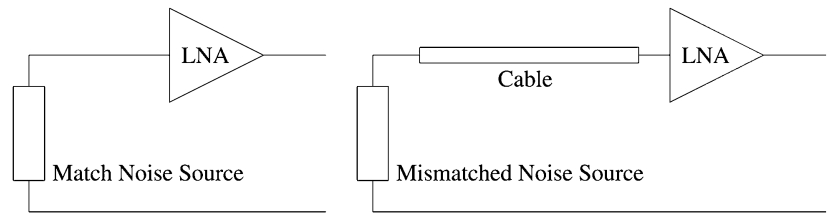

(a)

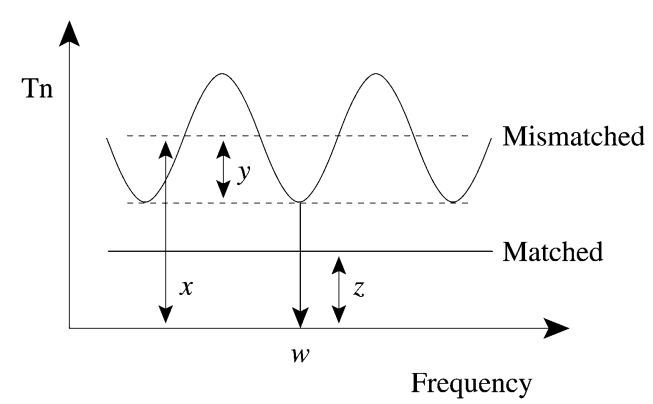

(b)

Fig. 1. Narrow-band frequency-variation method. (a) If the noise parameters of the LNA under test are all constant, then one matched and one mismatched noise temperatures can render the four noise parameters analytically. (b) If the mismatched generator impedance is $10 \Omega$ and the LNA has $T_{\min }=12 \mathrm{~K}$, $N=0.015$, and $Z_{\text {opt }}=(80-j 20) \Omega$, then $x=29.6 \mathrm{~K}, y=12.4 \mathrm{~K}$, and $z=13.4 \mathrm{~K}$. These $x, y, z$, and $w$, which is the frequency corresponds to the minimum mismatched noise temperature, can be used to calculate the four noise parameters.

Mathematically, the noise temperature can be expressed as

$$
\begin{aligned}
T_{n}=\left[T_{\min }+\right. & \left.4 T_{0} N \frac{\gamma_{g}^{2}+\gamma_{\mathrm{opt}}^{2}}{\left(1-\gamma_{g}^{2}\right)\left(1-\gamma_{\mathrm{opt}}^{2}\right)}\right] \\
& -\left[\frac{8 T_{0} N \gamma_{g} \gamma_{\mathrm{opt}}}{\left(1-\gamma_{g}^{2}\right)\left(1-\gamma_{\mathrm{opt}}^{2}\right)}\right] \cos \left(\theta_{g}-\theta_{\mathrm{opt}}\right) .
\end{aligned}
$$

If this $\Gamma_{g}\left(=\gamma_{g} \exp \left(j \theta_{g}\right)\right)$ is periodic and comes from a lossless input cable with length $L$, we can write

$$
\theta_{g}=\theta_{0}-\frac{4 \pi L}{v_{c}} f=\theta_{0}+m f
$$

where $v_{c}$ is the speed of light in the cable, and $m$ is as defined above. $T_{n}$ can now be simplified as

$$
\begin{aligned}
\text { matched } T_{n} & =z \mathrm{~K} \\
\text { mismatched } T_{n} & =x-y \cos \left(m f-\theta_{0}+\theta_{\text {opt }}\right) \mathrm{K} .
\end{aligned}
$$

Since the four unknowns $x, y, z$, and $\theta_{\text {opt }}$ can be directly inferred from the noise-temperature curves, noise parameters $\gamma_{\text {opt }}, N$, and $T_{\min }$ can be determined as follows:

$$
\begin{aligned}
\gamma_{\mathrm{opt}} & =\frac{x-z}{y \gamma_{g}}-\sqrt{\left(\frac{x-z}{y \gamma_{g}}\right)^{2}-1} \\
N & =y \frac{\left(1-\gamma_{g}^{2}\right)\left(1-\gamma_{\mathrm{opt}}^{2}\right)}{8 T_{0} \gamma_{g} \gamma_{\mathrm{opt}}} \\
T_{\min } & =\left(z-4 T_{0} N \frac{\gamma_{\mathrm{opt}}^{2}}{1-\gamma_{\mathrm{opt}}^{2}}\right) \mathrm{K} .
\end{aligned}
$$

Evidently, the mismatched measurement alone cannot render the four noise parameters. This is true even if the input cable in the mismatched measurement has characteristic impedance $Z_{\text {cable }}$ other than $50 \Omega$, as by setting $Z_{\text {cable }}$ to be the system's characteristic impedance. 


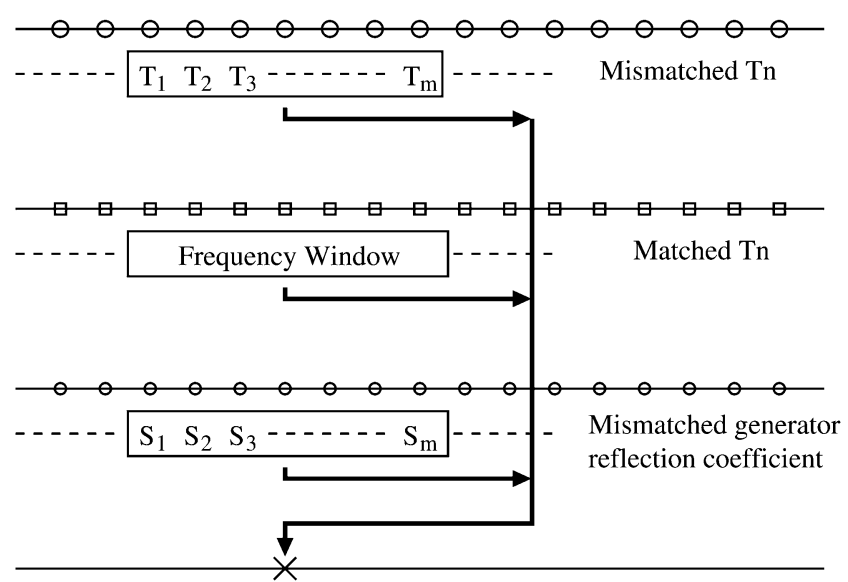

Noise parameters at this frequency point

Fig. 2. Wide-band frequency-variation method. The noise parameters at each frequency are calculated from the measured noise temperatures and reflection coefficients inside the frequency-sampling window. By moving this window along the frequency axis, the global noise parameters can be obtained. Here, $S_{i}\left(=\Gamma_{i}\right)$ is the mismatched generator reflection coefficient at the $i$ th frequency point.

\section{Numerical Wide-Band Frequency-Variation Method}

The idea of a narrow-band frequency-variation method has been partially implemented in the tuner method [10], where a long transmission line between the tuner and LNA under test results in a rapid change of $\Gamma_{g}$ within a small frequency interval. By assuming constant noise parameters in this interval, the adjacent noise-temperature information can reduce the number of tuner arrangements at each frequency and speed up the measurement procedure. As the resulting global noise parameters are now computed numerically in this mixed approach, one may wonder whether the frequency-variation method can be extended from narrow- to wide-band, analytical to numerical, and without the tuner.

With one matched and one mismatched $T_{n}$ measurement, a numerical wide-band frequency-variation method is proposed. If $\Gamma_{g}$ or, more specifically, its phase $\theta_{g}$, changes rapidly compared to the noise parameters of the LNA under test, the four noise parameters can be treated as locally constant within a frequency-sampling window that contains lots of frequency points. Applying the least-squares fit, the four unknowns can then be solved at the center frequency. By moving the frequency-sampling window point by point on the frequency axis and repeating the computation, the global noise parameters can be obtained (Fig. 2).

First, the relation between the generator reflection coefficients and noise temperatures needs to be arranged as

$\left[\begin{array}{c}T_{1} \\ T_{2} \\ \vdots \\ T_{m} \\ T_{50 \Omega}\end{array}\right]=\left[\begin{array}{cccc}1 & \frac{1}{\left(1-\gamma_{1}^{2}\right)} & \frac{\gamma_{1} \cos \left(\theta_{1}\right)}{\left(1-\gamma_{1}^{2}\right)} & \frac{\gamma_{1} \sin \left(\theta_{1}\right)}{\left(1-\gamma_{1}^{2}\right)} \\ 1 & \frac{1}{\left(1-\gamma_{2}^{2}\right)} & \frac{\gamma_{2} \cos \left(\theta_{2}\right)}{\left(1-\gamma_{2}^{2}\right)} & \frac{\gamma_{2} \sin \left(\theta_{2}\right)}{\left(1-\gamma_{2}^{2}\right)} \\ \vdots & \vdots & \vdots & \vdots \\ 1 & \frac{1}{\left(1-\gamma_{m}^{2}\right)} & \frac{\gamma_{m} \cos \left(\theta_{m}\right)}{\left(1-\gamma_{m}^{2}\right)} & \frac{\gamma_{m} \sin \left(\theta_{m}\right)}{\left(1-\gamma_{m}^{2}\right)} \\ 1 & 1 & 0 & 0\end{array}\right]\left[\begin{array}{l}a \\ b \\ c \\ d\end{array}\right]$ where $T_{i}$ for $i=1 \sim m$ is the noise temperature corresponding to the mismatched generator impedance $\Gamma_{i}\left(=\gamma_{i} \exp \left(j \theta_{i}\right)\right)$, and $T_{50 \Omega}$ is the matched noise temperature. When the matched reflection coefficient is not a perfect zero, as in reality, the last row of the above $[X]_{(m+1) \times 4}$ matrix can then be easily modified. If all the matched noise temperatures within the frequency-sampling window are used in the computation to enhance the accuracy, the size of the matrix $[X]$ becomes $2 m \times-4$. The intermediate matrix that requires the inverse operation, namely $\left([X]^{t}[X]\right)$, now has a dimension of $2 m \times 2 m$, rather than $(m+$ 1) $\times(m+1)$.

One way to reduce the size of the matrix $[X]$ and, therefore, the computation time, is to subtract at each frequency point the match $T_{n}$ from the mismatched one, i.e.,

$$
\Delta T_{n}=\left.T_{n}\right|_{\text {mismatched }}-\left.T_{n}\right|_{\text {matched }}
$$

and

$$
[\Delta T]=\left[\begin{array}{c}
\Delta T_{1} \\
\Delta T_{2} \\
\vdots \\
\Delta T_{m}
\end{array}\right]=[\Delta X]\left[\begin{array}{l}
b \\
c \\
d
\end{array}\right]
$$

where $[\Delta X]_{m \times 3}$ is a function of the matched and mismatched generator reflection coefficients. Therefore,

$$
\left[\begin{array}{l}
b \\
c \\
d
\end{array}\right]=\left([\Delta X]^{t}[\Delta X]\right)^{-1}[\Delta X]^{t}[\Delta T] .
$$

Once $b, c$, and $d$ are known, the remaining $a$ can be easily calculated from the matched $T_{n}$.

\section{WIDE-BAND FREQUENCY-VARIATION IMPLEMENTATION}

\section{A. Preliminary Simulation}

In the simulation (Fig. 3), the LNA under test has the following noise parameters:

$$
\begin{aligned}
T_{\min } & =\left(0.0067 f^{2}+0.0064 f+8.47\right) \mathrm{K} \\
R_{\mathrm{opt}} & =\left(0.29 f^{2}-10.100 f+107\right) \Omega \\
N & =\left(0.0068 f^{2}+0.0099 f+8.47\right) / 1000 \\
X_{\mathrm{opt}} & =(-4.53 f+80) \Omega
\end{aligned}
$$

where $f$ is the frequency in gigahertz, and $R_{\mathrm{opt}}+j X_{\mathrm{opt}}$ is the optimum generator impedance. The 50- $\Omega$ lossless air-dielectric $\left(\epsilon_{r}=1\right)$ cable between the 10- $\Omega$ noise source and LNA has length $L=7.5 \mathrm{~cm}$. The rectangular frequency-sampling window is chosen to be $2 \mathrm{GHz}$, which corresponds to a complete $2 \pi$ phase shift for $\Gamma_{g}$. With a 0.1-GHz frequency step, there are $1+2 / 0.1=21$ points within this window. The simulated results of the wide-band frequency-variation method show a global agreement with their theoretical counterparts. If the mismatched generator impedance in the simulation is changed from 10 to $20 \Omega$, similar simulated results can be obtained. If changed to $40 \Omega$, the computation goes divergent.

Thus far, both matched and mismatched noise measurements are needed to avoid the degeneracy of the matrix $\left([X]^{t}[X]\right)^{-1}$. 


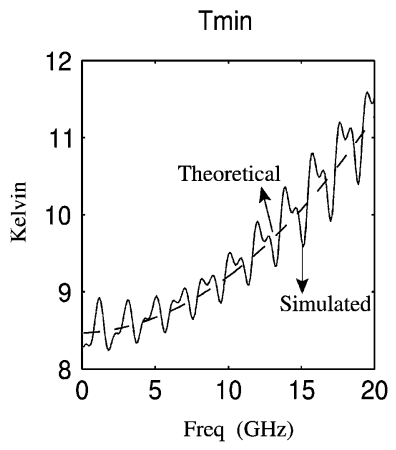

(a)

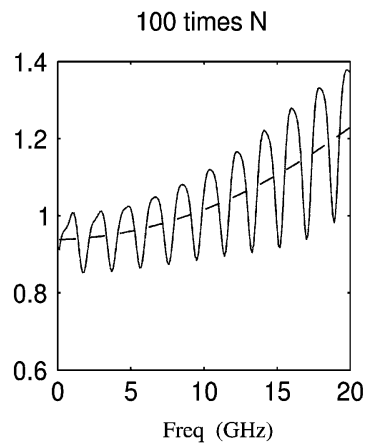

(b)

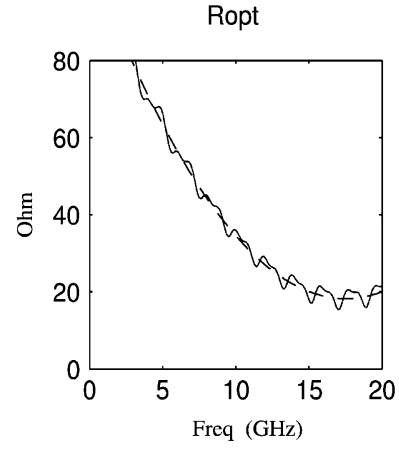

(c)

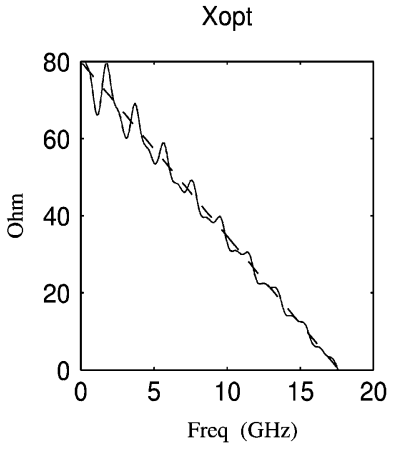

(d)

Fig. 3. Noise-parameter simulation using the wide-band frequency-variation method. Graphs (a)-(d) correspond to the minimum noise temperature, noise ratio, and the real and imaginary parts of the optimum generator impedance. In each graph, the dashed curve is the theoretical noise parameter of the LNA. The solid curve is the simulated result. The ripples on the solid curve are due to the rectangular frequency-sampling window used in the simulation, and can be removed by using a triangular frequency-sampling window.

An alternative to this two-step approach is to cascade two different cables, such as a $50-\Omega$ cable followed by a $10-\Omega$ cable of the same length; therefore, only one noise temperature measurement is required. While it is difficult to prove mathematically, computer simulation does generate nondivergent solutions. The drawback of this one-step approach, apparently, is the lack of the matched noise temperature information.

\section{B. Algorithm Optimization}

In the above simulation, the resulting noise-parameter curves are all embedded with ripples, which depend on the cable length and are worth further exploration. For simplicity, the magnitude of the generator reflection coefficient $\gamma_{g}\left(=\left|\Gamma_{g}\right|\right)$ is assumed to be constant, and the phase $\theta_{g}$ is $4 \pi L f / v_{c}$, where $v_{c}$ is the speed of light in the cable and $L$ is the cable length. By defining $W_{L}$ as $0.5 v_{c} / L$, the difference between the mismatched and matched noise temperatures at each frequency point is

$$
\triangle T_{n}=b^{\prime}+c^{\prime} \cos \left(\frac{2 \pi f}{W_{L}}\right)+d^{\prime} \sin \left(\frac{2 \pi f}{W_{L}}\right)
$$

with

$$
b^{\prime}=b \frac{\gamma_{g}}{1-\gamma_{g}^{2}} \quad c^{\prime}=c \frac{\gamma_{g}}{1-\gamma_{g}^{2}} \quad d^{\prime}=d \frac{\gamma_{g}}{1-\gamma_{g}^{2}} .
$$

If all the frequency points within the frequency-sampling window, which is chosen as a rectangle with base width $W_{L}$, are equally weighted, the use of least squares fit in the densely sampled case have solutions $b_{\mathrm{ls}}^{\prime}, c_{\mathrm{ls}}^{\prime}, d_{\mathrm{ls}}^{\prime}$ as follows:

$$
\begin{aligned}
b_{\mathrm{ls}}^{\prime} m & =\sum_{j=1}^{m} \Delta T_{i} \\
c_{\mathrm{ls}}^{\prime} \frac{m}{2} & =\sum_{j=1}^{m} \Delta T_{i} \cos \left(\frac{2 \pi f}{W_{L}}\right) \\
d_{\mathrm{ls}}^{\prime} \frac{m}{2} & =\sum_{j=1}^{m} \Delta T_{i} \sin \left(\frac{2 \pi f}{W_{L}}\right) .
\end{aligned}
$$

In terms of integration instead of summation, we obtain

$$
\begin{aligned}
b_{\mathrm{ls}}^{\prime} & =\frac{1}{W_{L}} \int_{f-W_{L} / 2}^{f+W_{L} / 2} \triangle T_{n}\left(f^{\prime}\right) d f^{\prime} \\
& =\frac{1}{W_{L}} \int_{-\infty}^{\infty}\left[\triangle T_{n}\left(f^{\prime}\right) \Pi\left(\frac{f^{\prime}-f}{W_{L}}\right)\right] d f^{\prime} \\
& =\frac{1}{W_{L}} \triangle T_{n}(f) \otimes \Pi\left(\frac{f}{W_{L}}\right) \\
c_{\mathrm{ls}}^{\prime} & =\frac{1}{W_{L}}\left[\triangle T_{n}(f) \cos \left(\frac{2 \pi f}{W_{L}}\right)\right] \otimes \Pi\left(\frac{f}{W_{L}}\right) \\
d_{\mathrm{ls}}^{\prime} & =\frac{1}{W_{L}}\left[\triangle T_{n}(f) \sin \left(\frac{2 \pi f}{W_{L}}\right)\right] \otimes \Pi\left(\frac{f}{W_{L}}\right)
\end{aligned}
$$

where $\otimes$ is convolution. Applying the Fourier transformation, while omitting the lengthy intermediate steps, the resulting spectral (Fourier) domain counterparts, as $f \rightarrow s$, are

$$
\begin{aligned}
\tilde{b}_{\mathrm{ls}}^{\prime}(s)= & \tilde{b}^{\prime}(s) \operatorname{sinc}\left(s W_{L}\right) \\
& +\frac{1}{2}\left[\tilde{c}^{\prime}\left(s-\frac{1}{W_{L}}\right)+\tilde{c}^{\prime}\left(s+\frac{1}{W_{L}}\right)\right] \operatorname{sinc}\left(s W_{L}\right) \\
& +\frac{1}{2}\left[\tilde{d}^{\prime}\left(s-\frac{1}{W_{L}}\right)-\tilde{d}^{\prime}\left(s+\frac{1}{W_{L}}\right)\right] \operatorname{sinc}\left(s W_{L}\right) \\
\tilde{c}_{\mathrm{ls}}^{\prime}(s)= & \tilde{c}^{\prime}(s) \operatorname{sinc}\left(s W_{L}\right) \\
& +\left[\tilde{b}^{\prime}\left(s-\frac{1}{W_{L}}\right)+\tilde{b}^{\prime}\left(s+\frac{1}{W_{L}}\right)\right] \operatorname{sinc}\left(s W_{L}\right) \\
& +\frac{1}{2}\left[\tilde{c}^{\prime}\left(s-\frac{2}{W_{L}}\right)+\tilde{c}^{\prime}\left(s+\frac{2}{W_{L}}\right)\right] \operatorname{sinc}\left(s W_{L}\right) \\
& +\frac{1}{2}\left[\tilde{d}^{\prime}\left(s-\frac{2}{W_{L}}\right)-\tilde{d}^{\prime}\left(s+\frac{2}{W_{L}}\right)\right] \operatorname{sinc}\left(s W_{L}\right) .
\end{aligned}
$$

A similar expression for $\tilde{d}_{\mathrm{ls}}^{\prime}$ also exists. In the spectral domain, it is obvious that $\Pi\left(f / W_{L}\right)$ can be treated as a filter $\operatorname{sinc}\left(s W_{L}\right)$ with nulls at $s=n / W_{L}$ to remove the unwanted harmonics. The ripples on the resulting noise parameters are simply the residual of those harmonics.

A better choice reducing the ripples is the use of $\operatorname{sinc}^{2}\left(s W_{L}\right)$ filter, i.e., a triangular $\Lambda\left(f / W_{L}\right)$ frequency-sampling window 


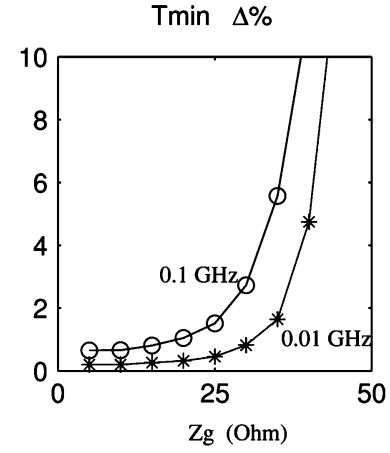

(a)

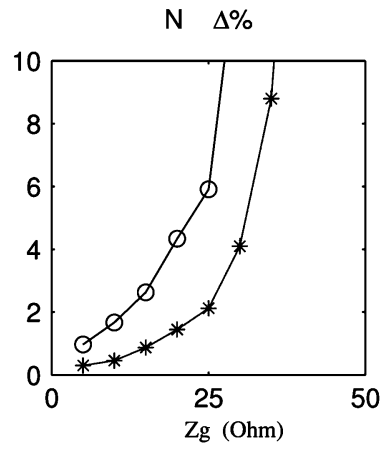

(b)

magnitude(Gamma_opt) $\quad \Delta \%$

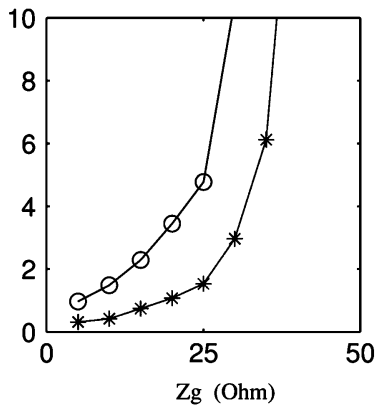

(c)
degree(Gamma_opt) $\Delta$

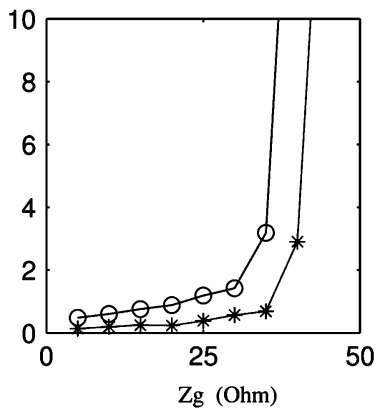

(d)

Fig. 4. Generator impedance $\left(Z_{g}\right)$ optimization for the wide-band frequency-variation method. Graphs (a)-(d) correspond to the resulting uncertainties of the minimum noise temperature, noise ratio, and magnitude and phase of the optimum reflection coefficient. In each graph, when the value of the mismatched $Z_{g}$ changes from 5 to $45 \Omega$, as on the $X$-axis, a $5 \% T_{n}$ uncertainty will have varying impact on the resulting noise parameter, indicated as percentage uncertainty on the $Y$-axis. In the simulation, the base of the triangular frequency-sampling window is $2 \mathrm{GHz}$, with a frequency step of 0.1 (circle curve) or $0.01 \mathrm{GHz}$ (asterisk curve). The air cable $\left(\epsilon_{r}=1\right)$ between the noise source and LNA has length $L=15 \mathrm{~cm}$, which corresponds to a complete $2 \pi$ phase change for $\Gamma_{g}$ in 1 GHz. The LNA in the simulation has $T_{\min }=10 \mathrm{~K}, N=0.01$, and $Z_{\text {opt }}=20 \Omega$.

with base width equal to $2 W_{L}$. With negligible harmonics present, the above expression can be revised as

$$
\begin{aligned}
& \tilde{b}_{\mathrm{ls}}^{\prime}(s)=\tilde{b}^{\prime}(s) \operatorname{sinc}^{2}(s W) \\
& \tilde{c}_{\mathrm{ls}}^{\prime}(s)=\tilde{c}^{\prime}(s) \operatorname{sinc}^{2}(s W) \\
& \tilde{d}_{\mathrm{ls}}^{\prime}(s)=\tilde{d}^{\prime}(s) \operatorname{sinc}^{2}(s W) .
\end{aligned}
$$

Since the errors are now from the nonunity main lobe of $\operatorname{sinc}^{2}\left(s W_{L}\right)$, it is not difficult to find the corresponding percentage errors in the derived noise parameters. Assume that it takes $10 \mathrm{GHz}$ for a complete $\Gamma_{\text {opt }}$ circle, i.e., $2 \pi$ phase change, on the Smith chart, and $1 \mathrm{GHz}$ for the $\Gamma_{g}$, the percentage errors of the resulting noise parameters will be in the range of $1-\operatorname{sinc}^{2}(1 / 10)=0.05$, i.e., $5 \%$. It will be $1 \%$ if the variation of $\Gamma_{\text {opt }}$ is 20 times slower than that of $\Gamma_{g}$.

\section{Choice of Generator Impedance}

The choice of $\Gamma_{g}$ itself has been extensively studied in applications using a tuner method [11]-[13]. With a crossed $\Gamma_{g}$ configuration, it is shown that a larger $\left|\Gamma_{g}\right|$ can have more accurate noise parameters if the $T_{n}$ uncertainty dominates; while a $\Gamma_{g} \approx \Gamma_{\text {opt }}$ is preferred if the uncertainty comes from the measured $\Gamma_{g}$. One may then ask which uncertainty prevails in the tunerless wide-band frequency-variation method.

One of the origins of $T_{n}$ uncertainties is the output power uncertainty of the noise source. Ambient temperature fluctuation is another factor, which affects not only the noise source, but also the noise contribution from the intermediate circuit between the noise source and device-under-test. The $\Gamma_{g}$ uncertainty can be attributed to the measurement error of the network analyzer, which may be due to the quality of the calibration kits or the network analyzer itself, and can be overcome to a large extent. The repeatability issue that is often associated with the worn mechanical tuners is no longer a problem here since there are no moving parts used to generate different $\Gamma_{g}$ points in this wide-band frequency-variation method. Therefore, only $T_{n}$ uncertainty has to be considered in the error analysis, and a highly mismatched generator impedance is preferred (Fig. 4).
In the simulation, the resulting noise-parameter uncertainties are smaller than those assigned on the noise temperature, which is because the final results at each frequency point are derived from many surrounding points within the frequency-sampling window, i.e., an averaging effect. Increasing the number of points within the frequency-sampling window can certainly reduce the resulting noise-parameter uncertainties, but with a diminishing marginal improvement. This averaging effect is one advantage of the wide-band frequency-variation method (over the tuner method) since it can be easily obtained.

Other considerations should also be taken into account when deciding the value of $\left|\Gamma_{g}\right|$. The first is whether the mismatched noise-temperature swing can be handled by the finite dynamic range of the noise measurement system. The other is whether a large $\left|\Gamma_{g}\right|$ will cause system instability, especially when measuring a transistor where the resulting output reflection coefficient of the transistor can be much larger than one. How to implement this large $\left|\Gamma_{g}\right|$ is another challenge. Empirically, $\left|\Gamma_{g}\right|=$ $0.7 \sim 0.8$ is feasible.

\section{Mismatched Circuit Implementation}

Since the output impedance of the commercial noise source is close to $50 \Omega$, an external mismatched circuit needs to be used for impedance transformation. The simplest way of making a mismatched circuit over a wide bandwidth is to use a resistor in series with the noise source. The drawback, however, is the degradation of the available noise power level. The larger the $\left|\Gamma_{g}\right|$ used, the smaller the equivalent excess noise ratio (ENR) will be. At $290-\mathrm{K}$ ambient temperature, the noise source's switch-off noise temperature is $T_{\text {cold }}=T_{0}$, while its switch-on noise temperature is $T_{\text {hot }}=290 \cdot 10^{(\mathrm{ENR} / 10)}+T_{0}$. Now with a reciprocal passive two-port circuit $[M]_{2 \times 2}$ connected to this noise source, the resulting $T_{\text {hot }}^{\prime}$ and $T_{\text {cold }}^{\prime}$ at the output of this two-port circuit can be derived from the conservation of noise power

$$
\begin{aligned}
\left(T_{\text {hot }}+T_{M}\right)\left|M_{21}\right|^{2} & =T_{\text {hot }}^{\prime}\left(1-\left|M_{22}\right|^{2}\right) \\
\left(T_{0}+T_{M}\right)\left|M_{21}\right|^{2} & =T_{\text {cold }}^{\prime}\left(1-\left|M_{22}\right|^{2}\right)
\end{aligned}
$$




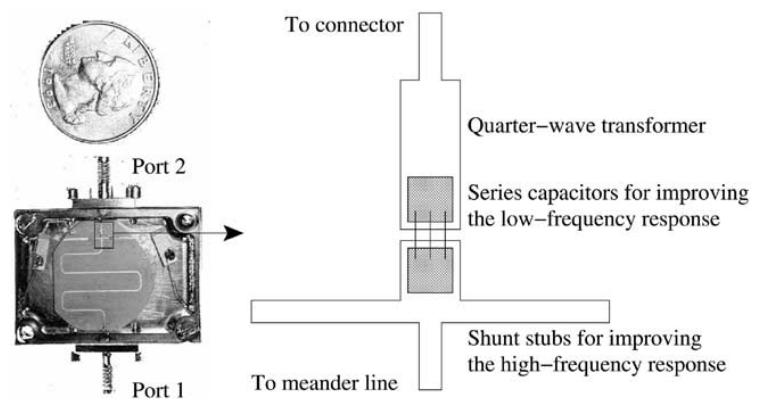

(a)

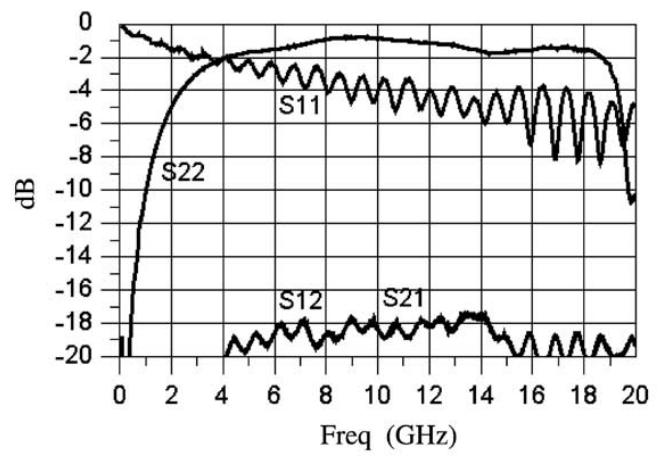

(b)

Fig. 5. Wide-band reactive mismatched circuit. (a) Since the quarter-wave transformer circuit is narrow-band, it needs to combine with serious capacitors and shunt stubs, as inductors, to render a wide-band mismatched reflection coefficient $\left(\Gamma_{g}\right)$. The meander line is included for a frequency-variation purpose. (b) $S$-parameters of the mismatched circuit. Originally, $S_{11}$ is designed as the $\Gamma_{g}$ for the wide-band noise-parameter measurement. However, the loss of the Duroid substrate and the imperfect connection between the connector and meander line causes this $S_{11}$ to be less useful. Instead, Port 2 is connected with a low-loss coaxial cable to generate the intended $\Gamma_{g}$ from $S_{22}$.

where $T_{M}$ is the matched noise temperature of the passive two-port circuit

$$
T_{M}=T_{0} \frac{1-\left|M_{22}\right|^{2}-\left|M_{21}\right|^{2}}{\left|M_{21}\right|^{2}}
$$

Since $T_{\text {cold }}^{\prime}$ equals to $T_{0}$, the modified ENR at the output of the two-port circuit is

$$
\begin{aligned}
\mathrm{ENR}^{\prime} & =10 \log _{10}\left(\frac{T_{\text {hot }}^{\prime}}{T_{0}}-1\right) \\
& =\mathrm{ENR}-10 \log _{10}\left(1+\frac{T_{M}}{T_{0}}\right) \\
& =\mathrm{ENR}-10 \log _{10}\left(\frac{1-\left|M_{22}\right|^{2}}{\left|M_{21}\right|^{2}}\right) \\
& \leq \mathrm{ENR} .
\end{aligned}
$$

A $200-\Omega$ series resistor, with $M_{21}=0.33$ and $M_{22}=-0.67$, has a matched noise temperature $T_{M}=4 T_{\mathrm{amb}}$. Now connecting this resistor to the output of a noise source with ENR = $5 \mathrm{~dB}$, we have $T_{\text {hot }}^{\prime}=490 \mathrm{~K}$ and $\mathrm{ENR}^{\prime}=-2 \mathrm{~dB}$. On the other hand, a lossless (reactive) circuit connected to the noise source can have $\mathrm{ENR}^{\prime}=\mathrm{ENR}$, as $1-\left|M_{22}\right|^{2}=\left|M_{21}\right|^{2}$ by definition. Now the question is whether a reactive mismatched circuit is allowed to have a broad bandwidth.

Since a lossless mismatched circuit can be treated as an impedance transformer, which changes the noise source's

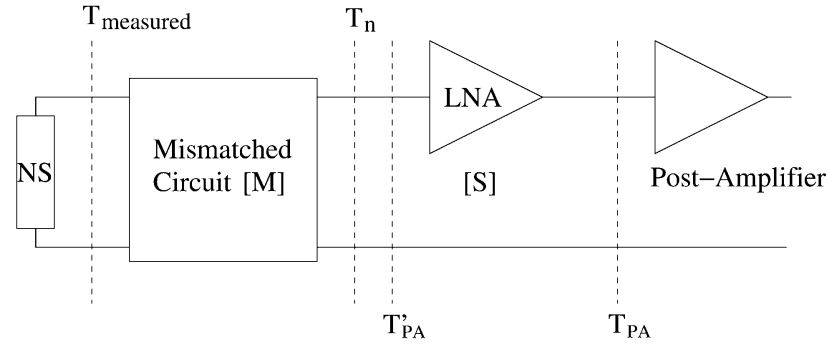

Fig. 6. Noise temperature deembedding. The measured noise temperature at the output reference plan of the noise source (NS) needs to be transformed to the input of LNA, i.e., $T_{\text {measured }} \rightarrow T_{n}$. To properly account for the noise from the post amplifier, there is $T_{\mathrm{PA}} \rightarrow T_{\mathrm{PA}}^{\prime}$. Noise temperature of the LNA is, therefore, $T_{n}-T_{\mathrm{PA}}^{\prime}$.

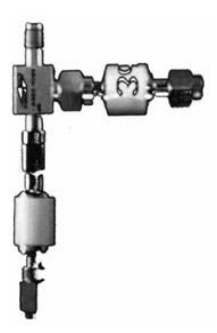

(a)

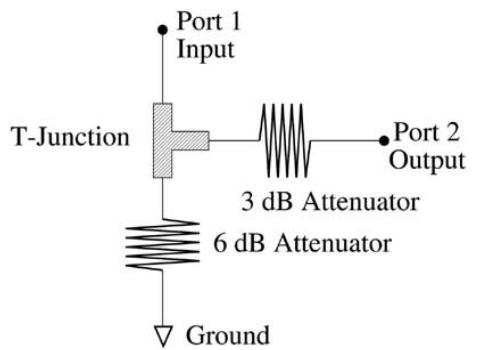

(b)
Fig. 7. Passive circuit used for the verification of wide-band frequency-variation method. (a) Photograph. (b) Schematic. The physical dimension of the T-junction causes both the $S$ - and noise parameters to be frequency-dependent.

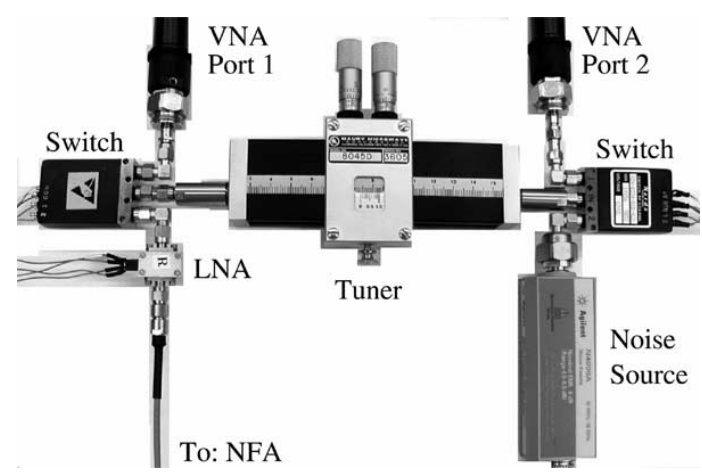

Fig. 8. Tuner method setup for noise-parameter measurement. The manual tuner MAURY 8045D uses two micrometers to change its output reflection coefficient, and one sliding bridge to provide a wide range of phase shift. The mechanical switches are NARDA 020-B234-A1B-4C0, which allow the alternative measurement of $S$-parameters and noise temperature. The noise source is Agilent N4000A with 6-dB ENR. The noise-figure analyzer (NFA) is Agilent N8975A. VNA is the Agilent vector network analyzer.

$50-\Omega$ output impedance to the intended generator impedance, its bandwidth, as bounded by the Bode-Fano criteria [14], should be infinite. Conceptually, this can be understood by imagining a very long lossless tapered line that transforms a $50-\Omega$ impedance to some other real impedance over a very large bandwidth.

A compact wide-band reactive mismatched circuit is, therefore, proposed (Fig. 5). Starting with a quarter-wave transformer, which is inherently narrow-band, series capacitors can then be added to boost the low-frequency reflection coefficient, and shunt stubs are included to generate the high-frequency mismatch. A long bond-wire connecting to a $50-\Omega$ resistor, and 


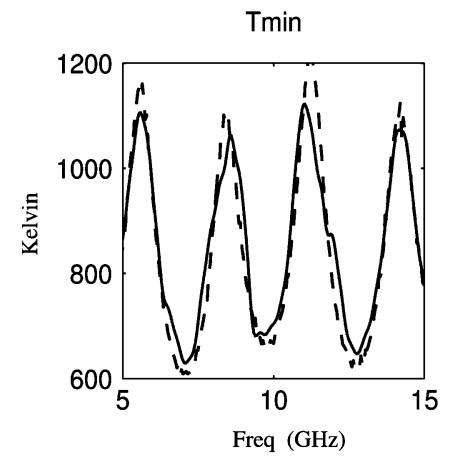

(a)

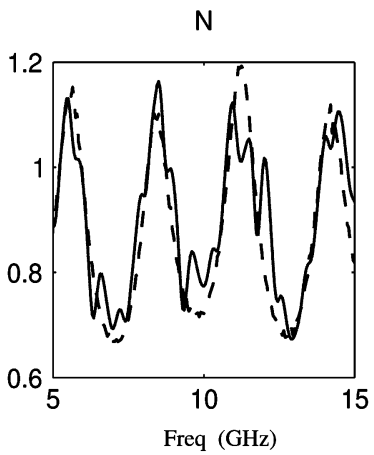

(b)

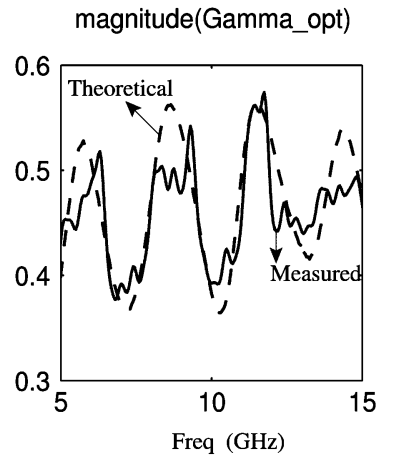

(c)

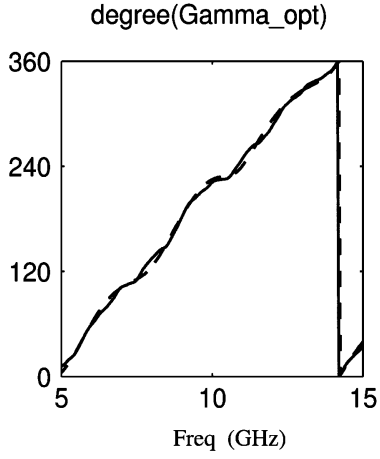

(d)

Fig. 9. Theoretical and measured noise parameters of the passive circuit. Graphs (a)-(d) correspond to the minimum noise temperature, noise ratio, and magnitude and phase of the optimum reflection coefficient. In each graph, the dashed curve is the theoretical noise parameter calculated from the $S$-parameters of the passive circuit. The solid curve is the noise parameter measured using the wide-band frequency-variation method.

then a capacitor, can again pull down the $\Gamma_{g}$ at the very low end of the frequency range to avoid potential oscillations when measuring a single transistor. DC bias/sense is also provided.

An intrinsic meander line is further included to generate the frequency variation where the $400-\mu \mathrm{m}$ line separation is wide enough to avoid signal coupling, as predicted from full-wave simulation. The whole circuit is fabricated on a soft 10-mil Duroid substrate. A thin silver sheet is heated to bond this substrate to a bronze chassis. A microwave absorber on the top plate is used to suppress the cavity modes. One concern with this mismatched circuit is the signal loss in the meander line; it means only a moderate value of $\left|\Gamma_{g}\right|$ can be obtained. The alternative is to use an external low-loss coaxial cable for frequency variation when a large $\left|\Gamma_{g}\right|$ is desired; the internal microstrip line in such a case is a thru line.

\section{VERIFICATION OF FREQUENCY-VARIATION METHOD}

\section{A. Noise-Temperature Deembedding}

In the mismatched noise-temperature measurement, the directly measured noise temperature $T_{\text {measured }}$ refers to the output reference plane of the noise source (Fig. 6). A deembedding procedure is then needed to shift the reference plane through the mismatched circuit $[M]_{2 \times 2}$. To better account for the nonzero $\Gamma_{g}$ of the noise source, the noise temperature $T_{M}$ of the mismatched circuit itself needs to be revised as follows:

$$
T_{M}=T_{\mathrm{amb}}\left(\frac{\left|1-\Gamma_{g} M_{11}\right|^{2}}{1-\left|\Gamma_{g}\right|^{2}} \frac{1-\left|M_{22}^{\prime}\right|^{2}}{\left|M_{21}\right|^{2}}-1\right)
$$

where $M_{22}^{\prime}$ is the output reflection coefficient of the mismatched circuit with the noise source in front of it as follows:

$$
M_{22}^{\prime}=M_{22}+M_{12} M_{22} \frac{\Gamma_{g}}{1-\Gamma_{g} M_{11}} .
$$

$T_{n}$ of the $[S]_{2 \times 2}$ LNA can now be calculated from the measured overall $T_{\text {measured }}$ as follows:

$$
\begin{aligned}
&\left(T_{\text {measured }}-T_{M}\right) \frac{1-\left|\Gamma_{g}\right|^{2}}{1-\left|\Gamma_{g} M_{11}\right|^{2}} \frac{\left|M_{21}\right|^{2}\left|S_{21}\right|^{2}}{\left|1-M_{22}^{\prime} S_{11}\right|^{2}} \\
&=T_{n} \frac{1-\left|M_{22}^{\prime}\right|^{2}}{\left|1-M_{22}^{\prime} S_{11}\right|^{2}}\left|S_{21}\right|^{2}
\end{aligned}
$$

i.e.,

$$
T_{n}=\left(T_{\text {measured }}-T_{M}\right) \frac{1-\left|\Gamma_{g}\right|^{2}}{\left|1-\Gamma_{g} M_{11}\right|^{2}} \frac{\left|M_{21}\right|^{2}}{1-\left|M_{22}^{\prime}\right|^{2}} .
$$

The other deembedding procedure concerns the second-stage (post-amplifier) noise contribution, which is negligible if the device-under-test is a high-gain LNA, but needs to be taken into account in the case of a passive circuit or a single transistor. With the noise parameters of the second-stage circuit known first, its noise temperature $T_{\mathrm{PA}}$ can be calculated from the designated generator reflection coefficient $\Gamma_{\mathrm{PA}}$, which is a function of the preceding $\Gamma_{g},[M]_{2 \times 2}$, and $[S]_{2 \times 2}$ as follows:

$$
\Gamma_{\mathrm{PA}}=S_{22}+\Gamma_{g} \frac{S_{12} S_{22}}{1-M_{22}^{\prime} S_{11}} \frac{M_{12} M_{21}}{1-\Gamma_{g} M_{11}} \Gamma_{g}
$$

where $[M]_{2 \times 2}$ will be replaced by an unitary matrix in the matched noise-temperature case. The transformed noise contribution $T_{\mathrm{PA}}^{\prime}$ at the input of the LNA will be

$$
T_{\mathrm{PA}}^{\prime}=T_{\mathrm{PA}} \frac{1-\left|\Gamma_{\mathrm{PA}}\right|^{2}}{\left|S_{21}\right|^{2}} .
$$

Once the matched and mismatched noise temperatures are available, the noise parameters can be derived using the wide-band frequency-variation method.

\section{B. Verification Using Passive Circuit and Conventional Tuner Method}

Since the noise parameters of a reciprocal passive circuit under thermal equilibrium can be calculated from its $S$-parameters [15], a reciprocal passive circuit can be used to check the validity of the wide-band frequency-variation method. After testing the 3- and 6-dB attenuators, which have frequency-independent $S$-parameters and noise parameters, a frequency-dependent passive circuit is then tried [see Fig. 7(a) and (b)].

On the other hand, since the tuner method is widely used in measuring room-temperature noise parameters, a comparison of the measured results using tuner and wide-band frequency-variation methods is worthwhile (Fig. 8). As the automatic (programmable) tuner was not available at the time of the mea- 


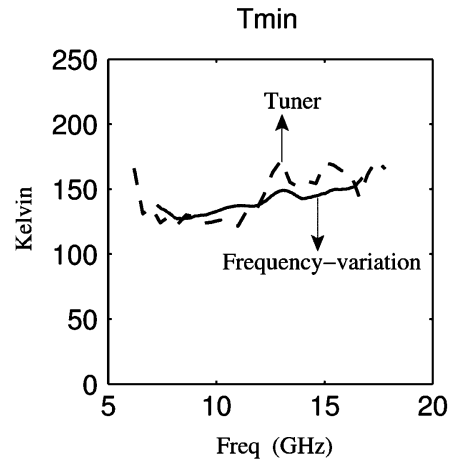

(a)

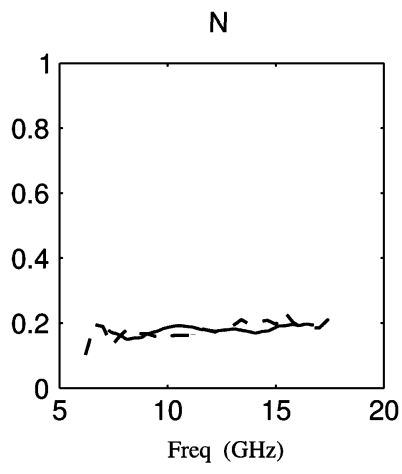

(b)
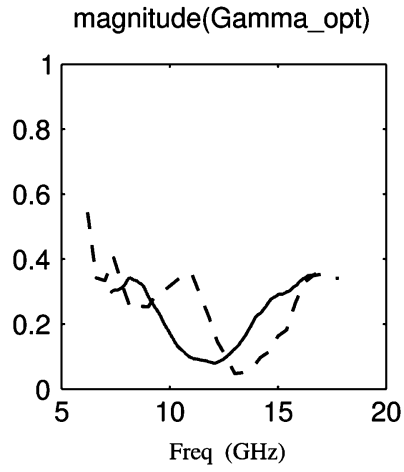

(c)

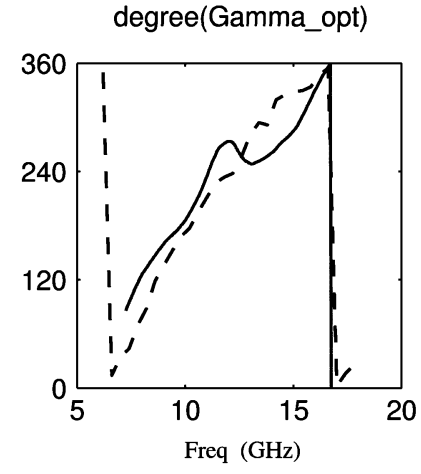

(d)

Fig. 10. Noise-parameter verification using tuner method. Graphs (a)-(d) correspond to the minimum noise temperature, noise ratio, and magnitude and phase of the optimum reflection coefficient. In each graph, the dashed curve is the noise parameter of a wide-band LNA measured using a tuner method with a 0.4-GHz frequency step. The solid curve is the noise parameter measured using the wide-band frequency-variation method with a $0.05-\mathrm{GHz}$ frequency step.

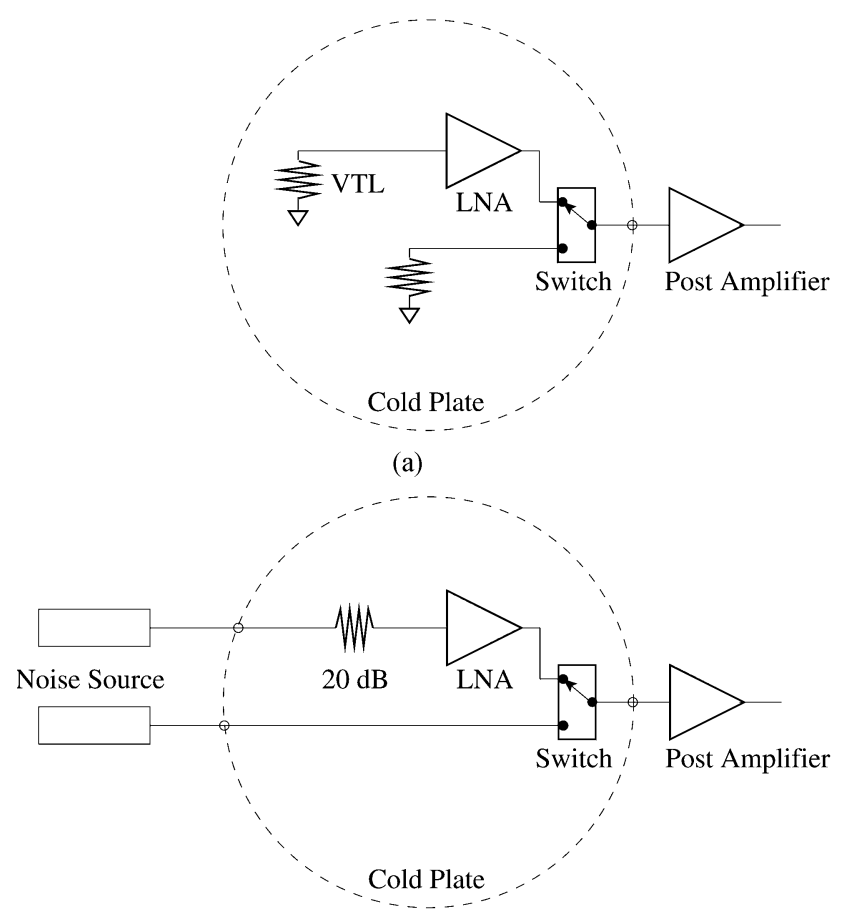

(b)

Fig. 11. Cryogenic noise-temperature measurement setups. (a) Variabletemperature-load method. The cryogenic noise temperature of the LNA under test is obtained by changing the physical temperature of a $50-\Omega$ resistive load (a coaxial termination), known as the variable-temperature load (VTL). The other branch, without the LNA, is used to measure the noise temperature of the post-amplifier for the calibration purpose. To reduce the temperature fluctuation, the heater and temperature sensor are both attached to the copper block in which the $50-\Omega$ resistive load is clamped. (b) Cold-attenuator method. The noise from a room-temperature commercial noise source is fed through the coaxial cable and the cold $20-\mathrm{dB}$ attenuator to the LNA. This 20-dB attenuator can reduce the power level of the incoming $T_{\text {hot }}$ and $T_{\text {cold }}$ to be comparable to that of the LNA. No attenuator is used on the calibration branch because of the much higher noise temperature of the room-temperature post-amplifier.

surement, a manual tuner setup is employed. In order to have a precise $S$-parameter measurement for each tuner setting, a pair of switches are used. Therefore, $S$-parameter measurement and noise-temperature measurement can be carried out alternatively. The signal-path difference in each switch was measured and taken into account in the noise-parameter calculation. The switch's phase repeatability has also been verified.

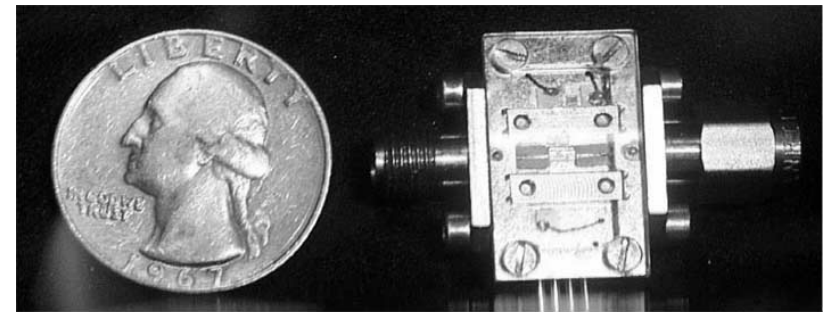

(a)

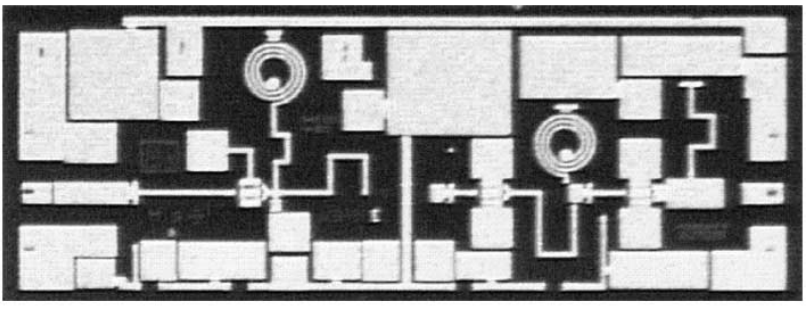

(b)

Fig. 12. Wide-band LNA used for noise-parameter measurement at different ambient temperatures. (a) Wide-band LNA. (b) MMIC chip inside. This MMIC has dimensions of $2000 \times 750 \times 75 \mu \mathrm{m}^{3}$ and is a three-stage circuit with signal input on the left.

The measured results of both the frequency-dependent passive circuit [see Fig. 9(a)-(d)] and the wide-band LNA [see Fig. 10(a)-(d)] illustrate that the wide-band frequency-variation method works well as room temperature, and as compared with the tuner method, the wide-band frequency-variation method takes much less time since its underlying principle is data sharing between adjacent frequency points. For measuring the noise parameters of a single transistor, an advanced automatic tuner setup with an adaptive algorithm for picking the designated reflection coefficients is probably the most desired [16], [17]. In the less stringent case, such as the wide-band LNA, the simple wide-band frequency-variation method works well.

\section{CRYOGENIC Wide-BAND NoISE MEASUREMENTS}

\section{A. Review of Noise-Temperature Measurement Systems}

There are two commonly used methods for measuring the noise temperature of a cryogenic microwave LNA. One is the so-called variable-temperature-load method, which can provide 


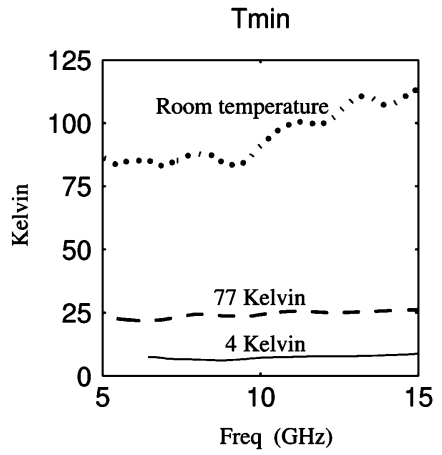

(a)

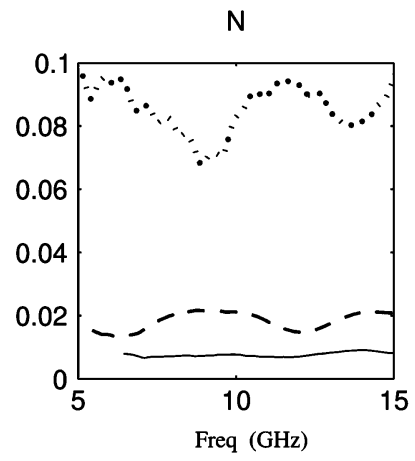

(b)
magnitude(Gamma_opt)

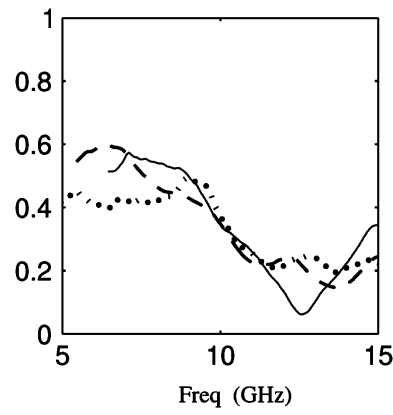

(c)
degree(Gamma_opt)

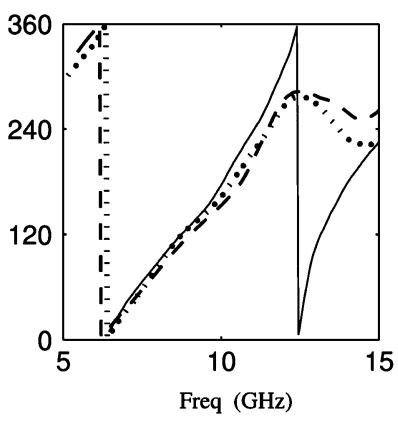

(d)

Fig. 13. Noise parameters of the wide-band LNA measured at different ambient temperatures. Graphs (a)-(d) correspond to the minimum noise temperature, noise ratio, and magnitude and phase of the optimum reflection coefficient. In each graph, the dotted curve is the noise parameter measured at room temperature, the dashed curve is at $77 \mathrm{~K}$, and the solid curve is at $4 \mathrm{~K}$.

a correct reading of the measurement, but is time consuming. The other is the cold-attenuator method, which is fast, but less accurate.

In the variable-temperature-load method [see Fig. 11(a)], the heater and temperature sensor are used to change and monitor the temperature of a $50-\Omega$ termination, and a coaxial cable is added in front of the LNA as a thermal buffer. Since this $50-\Omega$ resistor is clamped inside a copper block, the combined thermal constant is large enough to reduce the measured temperature fluctuations. After the post-amplifier, the noise power can be measured directly by a wide-band noise-figure analyzer, or down-converted using a wide-band mixer and then measured by a noise-figure meter. While the underlying physics of this method is straightforward, repeating the measurement is very time consuming because of the heating/cooling phases.

The other approach is the cold-attenuator method [see Fig. 11(b)], where the incoming noise power is generated by a commercial noise source. Since the noise source can be switched on and off rapidly, repeating the noise measurement will take much less time. When the noise source is turned off, its output noise temperature $T_{\text {cold }}$ will be close to room temperature. When it is on, the corresponding output noise temperature $T_{\text {hot }}$ can be calculated from the designated ENR value. For example, a noise source with 15-dB ENR has 9640-K output noise temperature. Since both $T_{\text {cold }}$ and $T_{\text {hot }}$ from the noise source are much larger than the noise temperature of the cryogenic LNA under test, a direct measurement tends to be erroneous. One way of overcoming this is to insert a cold 20-dB attenuator right in front of the LNA. This attenuator reduces the power level by a factor of 100 so the incoming noise power will be comparable to that of the LNA. Moreover, this cold $20-\mathrm{dB}$ attenuator provides a matched generator impedance to the LNA, and can also eliminate potential standing waves on the input cable.

However, the cold-attenuator method tends to be inaccurate because of the input cable and cold attenuator. As the input cable runs from room temperature to the cold plate, with possible heat sinks in between, it is difficult to precisely know this cable's noise contribution. The attenuator also runs the risk of being heated up. In contrast, there is no such problem in the variable-temperature-load method. A practical approach is to use the cold-attenuator method to find the optimum bias point for the LNA first, and then apply the variable-temperature-load method at known bias points to obtain the final results.

\section{B. Cryogenic Wide-Band Noise-Parameter Measurement}

The main obstacle in measuring the cryogenic wide-band noise parameters is that the room-temperature tuner is not compatible, at least conceptually, with the mentioned cryogenic noise measurement systems. In the variable-temperature-load method, the tuner needs to be located, apparently, inside the cryostat. In the cold-attenuator method, a room-temperature tuner connected to the noise source can generate a desired reflection coefficient, which, unfortunately, will become null after passing through the cold $20-\mathrm{dB}$ attenuator. If this cold $20-\mathrm{dB}$ attenuator is removed to allow a nonzero reflection coefficient presented to the cryogenic LNA, the measured results will be highly susceptible because the incoming noise temperatures $T_{\text {hot }}$ and $T_{\text {cold }}$ are now much larger then the noise temperature of the LNA. Therefore, in order to measure the cryogenic noise parameters, a complicated cryogenic tuner needs to be developed [18]-[20]. To circumvent the tuner idea, the proposed wide-band frequency-variation method is adopted here since it can be easily incorporated into the cryogenic noise measurement setups.

As in the room-temperature case, the cryogenic $S$-parameters of the mismatched circuit need to be measured first, which requires a prior two-port calibration inside the cryostat. This is done by putting each of the calibration standards into the cryostat in four consecutive thermal cycles, and measuring the corresponding $S$-parameters using a network analyzer, which is calibrated each time outside the cryostat. After applying the deembedding routine to shift the two-port reference planes into the cryostat, a cold $3-\mathrm{dB}$ attenuator is measured to confirm the calibration accuracy.

The amplifier under test is a wide-band LNA (Fig. 12), which has $25-\mathrm{dB}$ gain and $100-\mathrm{K}$ matched noise temperature at room temperature. Its cryogenic matched noise temperature is directly measured using the variable-temperature-load method. Its cryogenic mismatched noise temperature is obtained by inserting the wide-band mismatched circuit between the input cable and 
LNA. The four noise parameters of this LNA at different temperatures can then be derived using the wide-band frequencyvariation method (Fig. 13).

\section{CONCLUSION}

In this paper, a novel wide-band frequency-variation method for measuring noise parameters has been proposed. The underlying physics of this method is the use of matched and mismatched noise temperatures to provide the four measured values with which to solve for the four unknown noise parameters. Since noise parameters, in general, are functions of frequency, a numerical algorithm is adopted in this wide-band frequencyvariation method. The validity of this new method is confirmed by theoretical analyses and numerical simulations. As for the implementation, a mismatched module is designed to transform the $50-\Omega$ output impedance of the commercial noise source into that desired. Since this mismatched module can be easily incorporated into existing cryogenic noise-temperature measurement setups, cryogenic noise parameters are readily measured. As an example, the noise parameters of a wide-band LNA are measured at room temperature and 77 and $4 \mathrm{~K}$. This is the first time the global noise performance of a cryogenic wide-band LNA has been characterized.

\section{ACKNOWLEDGMENT}

Author R. Hu thanks Prof. G. Rebeiz, The University of Michigan at Ann Arbor, Dr. M. Edgar, D. Miller, F. Rice, G. Chattopadhyay, J. Kooi, and Prof. J. Zmuidzinas, all of the California Institute of Technology, Pasadena, Dr. J. Ward, Jet Propulsion Laboratory (JPL), Pasadena, CA, and Dr. F. Lo, National Radio Astronomy Observatory (NRAO), Charlottesville, $\mathrm{VA}$, for their support and encouragement.

\section{REFERENCES}

[1] S. Weinreb, "SIS mixer to HEMT amplifier optimum coupling network," IEEE Trans. Microwave Theory Tech., vol. MTT-35, pp. 1067-1069, Nov. 1987.

[2] S. Padin, D. P. Woody, J. A. Stern, H. G. Leduc, R. Blundell, C. Y. E. Tong, and M. W. Pospieszalski, "An integrated SIS mixer and HEMT IF amplifier," in 6th Int. Space Terahertz Technology Symp., Mar. 1995, pp. 134-139.

[3] E. F. Lauria, A. R. Kerr, M. W. Pospieszalski, S. K. Pan, J. E. Effland, and A. W. Lichtenberger, "A 200-300 GHz SIS mixer-preamplifier with $8 \mathrm{GHz}$ IF bandwidth," Nat. Radio Astronomy Observatory, Charlottesville, VA, ALMA Memo 378, June 2001.

[4] M. Sannino, "On the determination of device noise and gain parameters," Proc. IEEE, vol. 67, pp. 1364-1366, Sept. 1979.

[5] R. Q. Lane, "The determination of device noise parameters," Proc. IEEE, vol. 57, pp. 1461-1462, Aug. 1969.

[6] J. Lange, "Noise characterization of linear two-ports in terms of invariant parameters," IEEE J. Solid-State Circuits, vol. SSC-2, pp. 37-40, June 1967.

[7] G. Caruso and M. Sannino, "Computer-aided determination of microwave two-port noise parameters," IEEE Trans. Microwave Theory Tech., vol. MTT-26, pp. 639-642, Sept. 1978.

[8] R. P. Meys, "A wave approach to the noise properties of linear microwave devices," IEEE Trans. Microwave Theory Tech., vol. MTT-26, pp. 34-37, Jan. 1978.

[9] V. D. Larock and R. P. Meys, "Automatic noise temperature measurement through frequency variation," IEEE Trans. Microwave Theory Tech., vol. MTT-82, pp. 1286-1288, Aug. 1982.

[10] J. W. Archer and R. A. Batchelor, "Fully automatic on-wafer noise characterization of GaAs MESFET's and HEMT's," IEEE Trans. Microwave Theory Tech., vol. 40, pp. 209-216, Feb. 1992.
[11] S. Bosch and L. Martens, "Improved impedance-pattern generation for automatic noise-parameter determination," IEEE Trans. Microwave Theory Tech., vol. 46, pp. 1973-1678, Nov. 1998.

[12] S. Bosch and L. Martens, "Experimental verification of pattern selection for noise characterization," IEEE Trans. Microwave Theory Tech., vol. 48, pp. 156-158, Jan. 2000

[13] A. C. Davidson, B. W. Leake, and E. Strid, "Accuracy improvements in microwave noise parameter measurements," IEEE Trans. Microwave Theory Tech., vol. 37, pp. 1973-1978, Dec. 1989.

[14] D. M. Pozar, Microwave Engineering. Reading, MA: Addison-Wesley, 1990

[15] S. W. Wedge, "Computer-aided design of low noise microwave circuits," Ph.D. dissertation, Dept. Elect. Eng., California Inst. Technol., Pasadena, CA, 1991.

[16] M. Mitama and H. Katoh, "An improved computational method for noise parameter measurement," IEEE Trans. Microwave Theory Tech., vol. MTT-27, pp. 612-615, June 1979.

[17] L. Escotte, R. Plana, and J. Graffeuil, "Evaluation of noise parameter extraction methods," IEEE Trans. Microwave Theory Tech., vol. 41, pp. 382-387, Mar. 1993.

[18] M. W. Pospieszalski, "On the measurement of noise parameters of microwave two-port," IEEE Trans. Microwave Theory Tech., vol. MTT-34, pp. 456-458, June 1986.

[19] M. W. Pospieszalski, S. Weinreb, R. D. Norrod, and R. Harris, "FET's and HEMT's at cryogenic noise temperatures-Their properties and use in low-noise amplifiers," IEEE Trans. Microwave Theory Tech., vol. 36, pp. 552-560, Mar. 1988 .

[20] J. Laskar, J. J. Bautista, M. Nishimoto, M. Hamai, and R. Lai, "Development of accurate on-wafer, cryogenic characterization techniques," IEEE Trans. Microwave Theory Tech., vol. 44, pp. 1178-1183, July 1996.

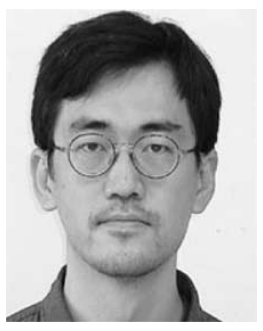

Robert (Shu-I) Hu received the B.S.E.E. degree from the National Taiwan University, Taipei, Taiwan, R.O.C., in 1990, and the Ph.D. degree from The University of Michigan at Ann Arbor, in 2003.

From 1996 to 1999, he was with the Academia Sinica, Taipei, Taiwan, R.O.C., where he was involved with millimeter-wave receivers. From 1999 to 2003, he was with the California Institute of Technology, Pasadena, where he was involved with millimeter-wave wide-band receivers. $\mathrm{He}$ is currently with Academia Sinica. His research interests include microwave and millimeter-wave electronics.

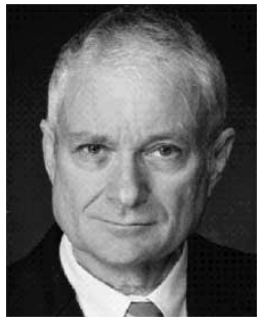

Sander Weinreb (S'56-M'63-SM'71-F'98-LF'02) received the B.S.E.E. and Ph.D. degrees from the Massachusetts Institute of Technology (MIT), Cambridge, in 1958 and 1963, respectively.

$\mathrm{He}$ is currently a Principal Scientist with the Jet Propulsion Laboratory (JPL), Pasadena, CA, and a Faculty Associate with the California Institute of Technology, Pasadena. Prior to this, he was a Research Professor with the Department of Physics and Astronomy at the University of Massachusetts. His main current area of research is the development of low-noise microwave and millimeter-wave integrated circuits (MMICs) for use in radio astronomy and atmospheric research. Prior to joining the University of Massachusetts, during 1989-1996, he was Principal Scientist and Leader of the Millimeter-Wave Design and Test Group at Martin Marietta Laboratories where he led the design of millimeter-wave MMICs and prototype radar and radiometer systems. In 1988 and 1989, he was a Visiting Professor with the University of Virginia. Prior to this, he was Head of the Electronics Division (1965-1985) and Assistant Director (1985-1988) with the National Radio Astronomy Observatory (NRAO), where he was responsible for the design, construction, operation, and maintenance of radio astronomy receivers at the Green Bank, WV, and Kitt Peak, AZ, observatories. While with the NRAO, he led the group responsible for the design of the electronics system for the very large array. He has authored over 120 publications in the areas of digital correlation techniques, radio-astronomy observations, array receivers, and LNAs. 